\title{
Directional Water Collection in Nanopore Networks
}

\author{
Rocio Gimenez, ${ }^{\dagger}$ Martín Gonzalo Bellino, ${ }^{*}{ }^{\dagger}$ (1) and Claudio Luis Alberto Berli**;(0) \\ †Instituto de Nanociencia y Nanotecnología CNEA-CONICET, Av. Gral. Paz 1499, San Martín, Buenos Aires B1650, Argentina \\ ${ }^{\ddagger}$ INTEC (Universidad Nacional del Litoral-CONICET) Predio CCT CONICET Santa Fe, RN 168, 3000, Santa Fe 1704, Argentina
}

Supporting Information

\begin{abstract}
The development of artificial nanosystems that mimic directional water-collecting ability of evolved biological surfaces is eagerly awaited. Here we report a new type of addressable water collection that is induced by coupling both vapor gradients, like a road drawn, and the temperature-tuned condensation in nanopores as step signals. What distinguishes the motion described here from the motions reported earlier is the fact that neither bulk liquid infiltration nor displacement of droplet is required. Instead, the motion results from a scanned water capture because of the temperature-dependent condensation command acting on the vapor pressure gradient track originated by a droplet without a bulk fluidic connection with a mesoporous film. This novel working principle demands only a small-range surface temperature control, which was entirely generated by a thermoelectric cell integrated to the mesoporous substrates. The strategy opens the route to achieving precise control over wetting location (from a few to hundreds of micrometers) and hence over the direction of water collected by these widely employed nanomaterials. Furthermore, as water is collected from condensation into the pores, the system naturally involves purification and subsequent delivery of clean water, which provides an added value to the proposed strategy.
\end{abstract}

\section{INTRODUCTION}

Notable biological surfaces on the micro- and nanometer scale, in both plant and animal kingdoms, display special features that control their interaction with water from humid air. ${ }^{1} \mathrm{~A}$ fascinating example is provided by certain beetles living in the desert that can capture water from the fog on their backs. ${ }^{2}$ Cactus species can also harvest water from humid air using exquisite multilevel surface structures. ${ }^{3}$ In addition to the adaptive characteristics that facilitate efficient fog capture, these adapted species present directional water collection as a notable ability of its biological structure. Mesoporous materials, which are composed of humidity-sensitive nanopores, enjoy a reputation as surfaces that collect water from humid air. ${ }^{4-6}$ Another interesting but less studied feature is the nanofluidic ability of mesoporous thin films (MTFs) to transport water by a bulk fluidic connection from drops deposited over its surface. ${ }^{7,8}$ Liquid from these sessile droplets infiltrates the porous matrix via capillary imbibition and builds a defined wetted region with a fluid front that advances in the mesoporous film. ${ }^{7}$ For instance, nanofluidic platforms based on MTF have been used to implement localized chemical reactions, ${ }^{9}$ for nanoflow manipulation, ${ }^{10}$ as a fluid sensor, ${ }^{11}$ and, notably, as an electrical current nanogenerator. ${ }^{12}$

Consider now a vapor gradient that influences a nanopore media. The resulting variations in relative humidity create a pathway for the directional collection of water. It is reasonable to expect that decreasing the temperature of the MTF can cause water capture along the pressure gradient. This scanned condensation of the water would be an innovative method for the creation of novel systems able to handle water uptake on the microscale. As proof of this concept, this work demonstrates that decreasing the temperature of the condensing surface drives a mobile wetting front onto the MTF guided from the vapor gradient associated with an adjacent water drop, which is not in direct contact with the patterned MTF. Given that water uptake produces a directional expansion of the wetted area, MTF exhibits an addressable water-collecting capability that is comparable to that found in evolved biological systems. A remarkable plus of the strategy is that it works under room conditions, which offers advantages in the development of practical devices. We further demonstrate the possibility to obtain and deliver purified water to micro-size nano-confined domains in a wellcontrolled fashion.

\section{RESULTS AND DISCUSSION}

Thick $(\approx 130 \mathrm{~nm})$ mesoporous silica thin films $(\approx 10 \mathrm{~nm}$ pore size) prepared as reported previously ${ }^{7,9}$ were patterned in defined millimeter-size shapes, using conventional photolithography techniques. ${ }^{13}$ Details are given in the Experimental Section and the process is schematically shown in Figure S1 (Supporting Information). The scanning electronic microscopy (SEM) images of the patterned mesoporous films elucidate its

Received: September 13, 2018

Accepted: November 16, 2018

Published: November 27, 2018 
structure (Figure 1). The system was operated in the lab atmosphere, under controlled conditions of temperature and

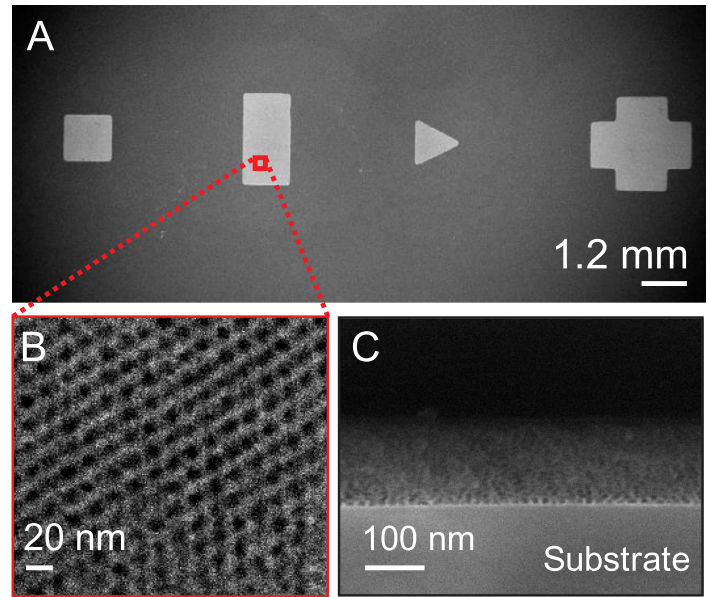

Figure 1. Microcopy images of the patterned MTF. (A) Lowmagnification SEM image taken from as-deposited films on silicon after photolithography patterning. (B) Magnified image that shows mesopores. (C) Cross-sectional view of the MTF.

relative humidity: $25{ }^{\circ} \mathrm{C}$ and $46 \%$, respectively. The water adsorption isotherm shown in Figure S2 (Supporting Information) reveals that in these ambient conditions the pores remain principally empty. The silicon substrate was then thermostated with a Peltier cell to homogeneously tune the temperature on the mesoporous film when a voltage is applied. When a water drop $(2 \mu \mathrm{L})$ was placed around $50 \mu \mathrm{m}$ beside the patterned MTF onto the silica substrate, a localized condensation region develops on the MTF surface. The wet area can be optically observed because water uptake produces a refractive index contrast in relation to the dry zone.

Figure 2 presents a schematic representation of the mechanisms controlling water collection through the mesoporous matrix. Water evaporation from the droplet surface creates a microclimate of increased vapor pressure $(p)$ in relation to the ambient vapor pressure $\left(p_{\mathrm{a}}\right)$, as schematically shown in Figure 2a. An estimation of the pressure profile can be made by using the classical description of droplet evaporation for a given temperature and without convective flows, where vapor distribution into the air is governed by molecular diffusion. ${ }^{14,15}$ From the characteristic diffusion time $t_{\mathrm{D}} \approx d^{2} / D$, where the diffusion coefficient of water vapor in air is $D=2810^{-6} \mathrm{~m}^{2} / \mathrm{s}$ at $25^{\circ} \mathrm{C}$, for example, the time for water molecules to diffuse a distance $d \approx 100 \mu \mathrm{m}$ is less than $1 \mathrm{~ms}$. According to this estimation, one may consider a quasi-steady state of vapor pressure because the diffusivity is practically instantaneous for small distances. In spherical coordinates, around a symmetric droplet of radius, $r_{\text {drop }}$, one has: $\partial\left(r^{2} \partial p /\right.$ $\partial r) / \partial r=0$, where $r$ is the radial coordinate. ${ }^{16}$ The boundaries of this equation are the saturated vapor pressure on the drop surface, $p_{\text {sat }}=p\left(r=r_{\text {drop }}\right)$, and the ambient pressure at long distances, $p_{\mathrm{a}}=p(r \rightarrow \infty)$. A straightforward integration of the governing equation yields

$$
p(r)=\left(p_{\text {sat }}-p_{\mathrm{a}}\right) \frac{r_{\text {drop }}}{r}+p_{\mathrm{a}}
$$

which describes pressure variation in the radial outward direction of the surrounding air. Equivalently, a gradient of relative humidity $\left(\mathrm{RH}=p / p_{\text {sat }}\right)$ develops around the droplet,

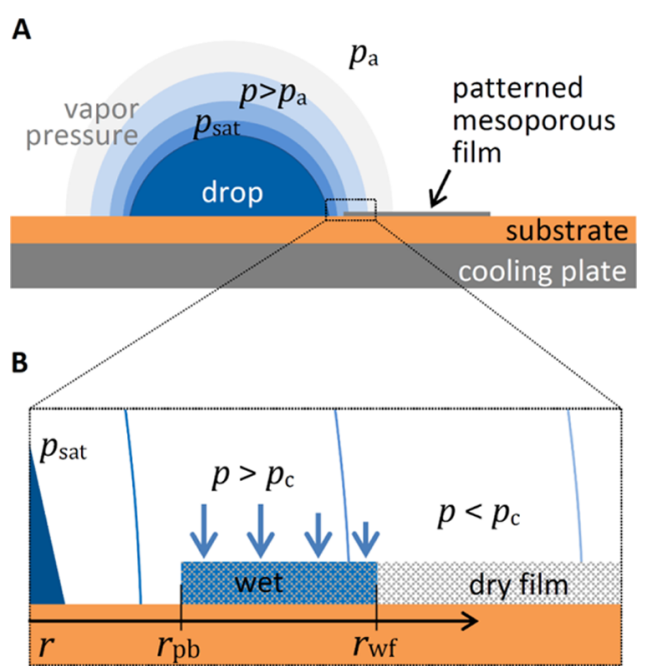

Figure 2. Scheme of the water-collecting system. (A) Schematic representation of vapor pressure contours around a sessile drop on a silicon substrate (side view); the drop border is micrometers apart from a region of patterned mesoporous film. The cooling plate of the thermoelectric cell in direct contact with the substrate is also shown. (B) Zoom of the mesoporous film in close vicinity of the drop border (side view). The blue arrows indicate the condensation flux, which takes place in the zone where the vapor pressure $(p)$ is higher than the condensation pressure $\left(p_{\mathrm{a}}\right)$ at the nanopores. The black arrow is the radial coordinate $(r)$ in the plane of the substrate, where $r_{\mathrm{pb}}$ is the location of the patterning border and $r_{\mathrm{wf}}$ is the position of the wetting front at a given temperature.

which decreases from $100 \%$ on the drop surface to $46 \%$ clear of the drop (room value). ${ }^{17,18}$ This microenvironment of enriched humidity spans over the patterned MTF and induces water condensation into the mesoporous matrix (Figure $2 b$ ).

Nanopore structures effectively capture water from unsaturated vapor, as the capillary pressure $\left(P_{\text {cap }}\right)$ arising from small curvatures in the nanoscale confinement strongly decreases the vapor pressure required for condensation $\left(p_{c}\right)$, in comparison to the condensation pressure on a flat surface $\left(p_{\text {sat }}\right) .{ }^{19}$ In fact, according to Kelvin-Laplace equation, the condensation pressure can be written as ${ }^{20}$

$$
p_{\mathrm{c}}(T)=p_{\text {sat }}(T) \exp \left(\frac{-P_{\mathrm{cap}} V_{\mathrm{m}}}{R T_{\mathrm{abs}}}\right)
$$

where $V_{\mathrm{m}}$ is molar volume of the liquid, $R$ is the gas constant, and $T_{\mathrm{abs}}$ is the absolute temperature. It is worth reminding that $p_{\text {sat }}(T)$ is a well-known function for water, ${ }^{21}$ which increases from 2.34 to $5.63 \mathrm{kPa}$ in the range $20-35{ }^{\circ} \mathrm{C}$. On the other hand, the air in direct contact with the patterned film has a decreasing vapor pressure, as shown in Figure $2 \mathrm{~b}$. Thus, water uptake into the mesoporous film occurs in the close vicinity of the droplet border, where $p \geq p_{c}$. Beyond a certain radial distance, where the vapor pressure is lower than $p_{\mathcal{c}}$, the film remains dry. At a given temperature, the wetting front reaches a steady position $\left(r_{\text {wf }}\right)$, as shown in Figure $2 \mathrm{~b}$.

The series of pictures in Figure 3a display how the wetted region grows as the substrate temperature decreases (cooling from 30.6 to $21.4{ }^{\circ} \mathrm{C}$ in this example). The dynamics of this directional water collection can be vividly observed on the Movie S1 (Supporting Information) accelerated by a factor of about 4. As mentioned above, when MTF was exposed to vapor gradient surrounding a droplet, water started to 


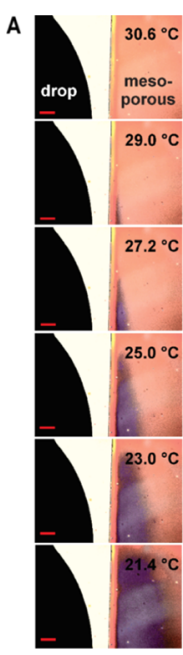

B

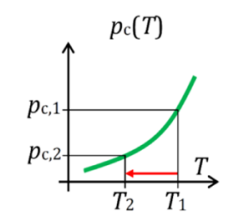

C
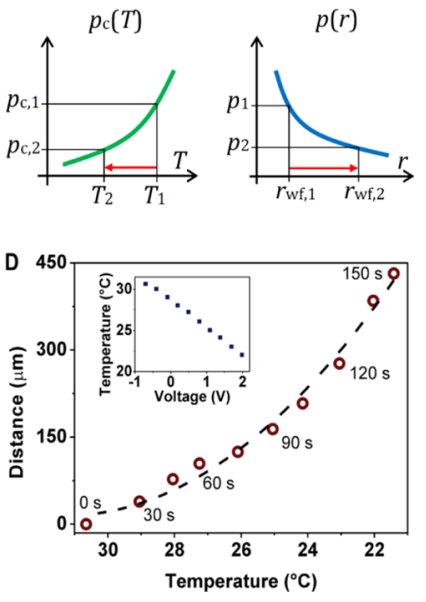

Figure 3. Working principle of the water-collecting system. (A) Timelapse optical microscopy sequence showing the advancement of the wetting front along the mesoporous film during a gradual temperature diminution. Scale bar $=50 \mu \mathrm{m}$. (B) Qualitative graph of the condensation pressure as a function of film temperature according to eq 2. (C) Qualitative graph of the vapor pressure as a function of radial distance, according to eq 1 . The red arrow indicates the displacement of the wetting front when the film temperature decreases. (D) Position of the wetting front as a function of substrate temperature; the distance was measured from the MTF patterning border, and corresponds to $r_{\mathrm{wf}}(T)-d_{0}$, where the distance $d_{0}$ is about $r_{\mathrm{pb}}$ (Figure $2 \mathrm{~b}$ ). The dashed line is to guide the eye (see Figure 4 for model prediction). Substrate temperature was controlled with a thermoelectric cell, where voltage varied from -1 to $2 \mathrm{~V}$ in steps of $0.02 \mathrm{~V} \mathrm{~s}^{-1}$. The inset shows the linear relation between the temperature measured at the substrate surface and the input voltages.

condense selectively on the mesoporous film and the position of the wetting front was displaced with relatively small temperature variations. In this process, the nanopores serve as temperature-activated condensing sites to be able to move the wetting front directionally. Imbibition driven by capillary condensation in nanopores has been recently demonstrated by theory and experiments, ${ }^{19}$ where vapor pressure was varied in a chamber at a given temperature. However, as a novel approach, our strategy consists in exploiting the natural vapor pressure gradient generated by water droplets at room conditions, whereas condensation is induced by controlling the surface temperature, thus additionally allowing localized and directional water uptake. In what follows, we describe this effect by using a qualitative analysis of the underlying phenomena of vapor transport.

The Kelvin-Laplace equation allows one to rationalize the thermal effect, taking into account that the condensation pressure increases with temperature (see eq 2 ) because of the dependence of both $p_{\text {sat }}(T)$ and the exponential factor (energy associated with the curvature); the overall effect is depicted in Figure $3 \mathrm{~b}$. Therefore, directly decreasing the film temperature enables condensation at lower vapor pressures, that is, in the outer regions of the gradient, which produces a displacement of the wetting front in the outward radial direction, as illustrated in Figure 3c (see also eq 1). Furthermore, assuming that the wetting front is defined by the condition $p\left(r_{\mathrm{wf}}\right) \approx$ $p_{\mathrm{c}}(T)$, one may infer the existence of an implicit function, $r_{\text {wf }}(T)$, connecting the diagrams of Figure 3b,c. Effectively, the function $r_{\mathrm{wf}}(T)$ was experimentally measured and is reported in
Figure 3d, where the dashed line is just to guide the eye. In what follows we explore the theory for such correlation.

In fact, equalizing eqs 1 and 2 yields

$$
\frac{r_{\mathrm{wf}}(T)}{r_{\mathrm{drop}}}=\frac{p_{\text {sat }}(25)-p_{\mathrm{a}}(25)}{p_{\text {sat }}(T) \exp \left[\frac{-2 \sigma V_{\mathrm{m}}}{r_{\mathrm{p}} R(273+T)}\right]-p_{\mathrm{a}}(25)}
$$

where the pressure values taken at $25{ }^{\circ} \mathrm{C}$ come from eq 1 and correspond to the vapor pressure in the air, which is supposed to be kept at ambient conditions during the experiments, as a first approximation. Also in eq 3, the capillary pressure has been included as $P_{\text {cap }}=2 \sigma / r_{\mathrm{p}}$, where $r_{\mathrm{p}}$ is the average pore radius and $\sigma$ is the water surface tension (this parameter slightly varies with temperature and may also be considered constant in the range $20-35^{\circ} \mathrm{C}$ ).

In order to compare the model prediction against experimental data, it should be taken into consideration that the distance plotted in Figure $3 \mathrm{~d}$ is $d(T)=r_{\mathrm{wf}}(T)-d_{0}$, where $d_{0}$ is an arbitrary constant that measures the initial position of the wetting front during image analysis. Here we left $d_{0}$ as a free parameter, together with the drop radius, $r_{\text {drop }}$, that enters eq 3 , which is also an effective value considering the oversimplifications made to derive eq 1 . Finally, the comparison is made in Figure 4, where it is readily seen that

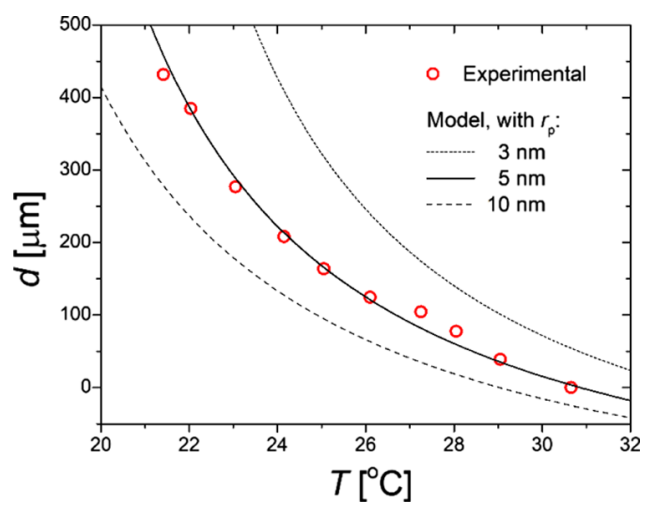

Figure 4. Theoretical background for the working principle. Wettingfront position as a function of temperature, for different pore radius. The distance plotted is $d=r_{\mathrm{wf}}(T)-d_{0}$, where $r_{\mathrm{wf}}(T)$ is included from eq 3. Parameter values used in calculations are: $\sigma=73 \mathrm{mN} \mathrm{m}^{-1}, V_{\mathrm{m}}=$ $18 \mathrm{~mL}, R=8.314 \mathrm{~J} \mathrm{~mol}^{-1} \mathrm{~K}^{-1}, p_{\text {sat }}(25)=3.17 \mathrm{kPa}$, and $p_{\mathrm{a}}(25)=$ $0.46 p_{\text {sat }}(25)$. The function $p_{\text {sat }}(T)$ was included as a correlation for the range $20-35{ }^{\circ} \mathrm{C}^{21}$ Fitting parameters are $r_{\text {drop }}=220 \mu \mathrm{m}$ and $d_{0}=175$ $\mu \mathrm{m}$ (see text for details).

the theoretical prediction closely follows the experimental trend. It is worth to remark that the model includes all the characteristic constants of water (reported in the caption of Figure 4) and the average pore radius of the mesoporous film, $r_{\mathrm{p}}=5 \mathrm{~nm}$. The only fitting parameters were $r_{\text {drop }}=220 \mu \mathrm{m}$ and $d_{0}=175 \mu \mathrm{m}$, whose values are quite reasonable for the experimental set.

In Figure 4, the dashed lines correspond to smaller and larger pore radius ( 3 and $10 \mathrm{~nm}$, respectively), for the same $r_{\text {drop }}$ and $d_{0}$ values. These curves illustrate the response of the system for mesoporous films with different pore size. For example, for a given temperature, smaller pore radius yields larger wetting fronts, which is because smaller radius increases capillary condensation, and thus, the system may capture water at relatively lower vapor pressures. Therefore, one may observe 
that eq 3 establishes a strong correlation between the wettingfront position and the surface temperature, which is fully based on physical parameters of the system.

The practical interest of this correlation is that the position of the wetting front can be handled by controlling the mesoporous film temperature, which is carried out by using a Peltier cooling system in our experiments. Liquid flow because of capillary action may also play a role, driving water from wet to dry zones into the film; however, the infiltration dynamics through the nanopore structure is very slow in comparison to condensation, ${ }^{19}$ whereas here the wetting front instantaneously followed the temperature changes. At this point, it is worth emphasizing that the film becomes wetted without a bulk fluidic connection with the droplet, in contrast to previous setups where MTF were infiltrated from liquid reservoirs. ${ }^{10}$

Considering a top view of the water-uptake phenomenon, such as in Figure 3a and Movie S1, it is readily seen how the $2 \mathrm{D}$ shape of the wetting front closely follows the isobar lines of the vapor pressure gradient. For a given film temperature, condensation occurs at radial positions $r \leq r_{\text {wf }}$, where $p(r) \leq$ $p_{\mathrm{c}}(T)$. Decreasing the film temperature produces an expansion of the wetted area, and the advance of the wetting front resembles a scanning of the peripheral isobars in the plane of the substrate. The effect is clearly observed because optical reflectance locally changes upon water saturation, providing a net contrast between wet and dry zones of the film.? Furthermore, it has been recently shown that the relationship between the amount of adsorbed water and light reflectance is rather linear. ${ }^{19}$ Control experiments performed in droplet absence showed no directional water collection (see Figure S3; Supporting Information), reinforcing the hypothesis that both components of the system (mesoporous matrix and water reservoir in noncontact mode) are necessary to produce an addressable water-uptake location.

In order to test the potential utility of the addressable nanocondensator to collect and deliver purified water, we conducted chemical reaction experiments on patterned MTF, in which reagents were supplied from sessile droplets. Two differing configurations were tested: droplets directly in contact with the MTF and droplets adjacent to the patterned region without fluidic contact with the MTF. Figure 5a shows the localized formation of $\mathrm{AgCl}_{(\mathrm{s})}$ when droplets of $0.1 \mathrm{M}$ silver nitrate $\left(\mathrm{AgNO}_{3}\right)$ and $1 \mathrm{M}$ sodium chloride $(\mathrm{NaCl})$ solutions are deposited over the opposite borders of a rectangular (1.2 $\mathrm{mm} \times 2.4 \mathrm{~mm})$ MTF patterning. ${ }^{9}$ Wetted regions grew from both drops via capillary imbibition, and the transported reagents produced an abrupt precipitation of silver chloride in the contact interface. ${ }^{9}$ In contrast, no precipitation was observed when droplets were not in touch with the MTF patterning (Figure 5b). This expected result makes evident the distillated nature of the collected water and simultaneously reveals an attractive strategy for malleable water purification on the microscale, further suggesting the potential to integrate this controlled supply of clean water into advanced microdevices. Although it can be argued that the instability of MTFs in aqueous media represents a liability of this system for longterm applications, ${ }^{22}$ our experiments show that they are usable at least within a reasonable time frame.

\section{CONCLUSIONS}

The development of systems that operate on "open pit" scenarios to provide addressable water collection, which is intrinsic to evolved natural systems, is becoming a key
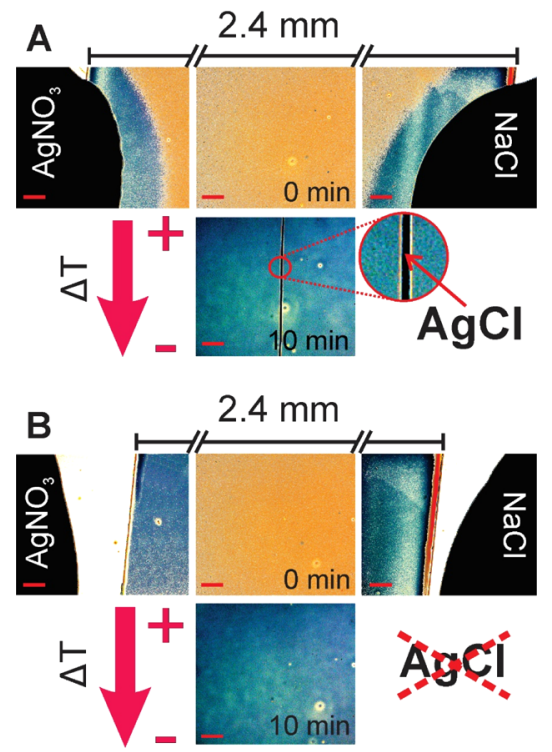

Figure 5. Purified water collector. Optical microscopy images of the region where the wetting fronts from different drops met. Drops of 0.1 $\mathrm{M} \mathrm{AgNO}_{3}$ and $1 \mathrm{M} \mathrm{NaCl}$ solutions were deposited in opposite sides of a patterned MTF with (A) or without (B) direct connection to the film. In both cases, the lower panels show the final stage (after decreasing temperature): as expected, silver chloride precipitation takes place only if there is a bulk fluidic connection between the drops and the MTF. Scale bar $=50 \mu \mathrm{m}$.

requirement for versatile devices, presenting a significant scientific and engineering challenge. Whereas studies of water collection into pore media in the past used pressure chambers at a given temperature, we have here explored the surface temperature-actuated condensation in a natural vapor gradient guide generated by water droplets under room conditions as the driving forces. Water-collecting directionality in the mesoporous films is the result of the condensation features tuned by surface cooling on drop vapor environment at ambient conditions. We have demonstrated that mesoporous films not only yield water condensation but also enable a directional water collection from the humidity of small droplets through relatively small MTF temperature variations. The temperature control has been established by voltage inputs, which offer additional opportunities for the development of microdevices with integrated smart water collection capabilities. Unlike the nanofluidic platforms based on capillary infiltration reported at present, in this noncontact mode the motion of the wetting front is fully driven by water uptake from the vapor phase. This strategy takes advantage of coupling both the vapor gradient around sessile drops, like road drawn, and the temperature-dependent condensation pressure in nanopores as step signals. We anticipate that this route can be extended readily to other nanoporous structures (nanostructured porous silicon, ${ }^{23}$ metal-organic frameworks, ${ }^{24}$ and so forth) to guide their water uptake. This new working principle opens the way for nanopore networks to be used as an accurately controllable wetting location versatile system and hence to direct the water collection. We therefore envision that this study will aid the development of novel addressable systems for water collection and purification. Owing to its high modularity and scalability, this self-purifying water supply may find applications in advanced microdevices. 


\section{EXPERIMENTAL SECTION}

Silicon wafers $\left\langle\begin{array}{lll}1 & 0 & 0\end{array}\right\rangle$ were cleaned with Milli-Q water, acetone, and isopropanol, and dried under nitrogen flow. The pattern to be transferred was defined by a conventional lithography technique. The fabrication starts with the spinning of a sacrificial AZ 2070 resin layer at $3000 \mathrm{rpm}$ (thickness 7 $\mu \mathrm{m})$ on which UV lithography was performed for $10 \mathrm{~s}$, and then developed. A $200 \mathrm{~nm}$ copper film was then deposited by sputtering with a subsequent lift-off with acetone. For the mesoporous film deposition step, the copper-patterned substrate was submerged in a silica precursor solution. Tetraethyl orthosilicate (TEOS) (Sigma-Aldrich) was used as the inorganic precursor, and Pluronics F127 (HO$\left.\left(\mathrm{CH}_{2} \mathrm{CH}_{2} \mathrm{O}\right)_{106}\left(\mathrm{CH}_{2} \mathrm{CH}\left(\mathrm{CH}_{3}\right) \mathrm{O}\right)_{70}-\left(\mathrm{CH}_{2} \mathrm{CH}_{2} \mathrm{O}\right)_{106} \mathrm{OH}\right)$ (Sigma-Aldrich) was used as the template. TEOS was prehydrolyzed by refluxing for $1 \mathrm{~h}$ in an acidic water $(\mathrm{HCl}$ $0.14 \mathrm{M})$ and ethanol $(\mathrm{EtOH})$ solution in the following ratios: $\left[\mathrm{H}_{2} \mathrm{O}\right] /[\mathrm{Si}]=1 ;[\mathrm{EtOH}] /[\mathrm{TEOS}]=5$. Next, Pluronics F127, ethanol, and acidic water $(\mathrm{HCl} 0.055 \mathrm{M})$ were added to the prehydrolyzed solution in order to prepare the precursor solution with final composition TEOS/EtOH/ $\mathrm{H}_{2} \mathrm{O} / \mathrm{F} 127$ equal to 1:40:5:0.0075 mol ratio. Mesoporous silica films were deposited by dip-coating at $2 \mathrm{~mm} / \mathrm{s}$ at $\mathrm{RH}$ of $35 \%$. After deposition, the samples were placed in a $50 \% \mathrm{RH}$ chamber overnight, and then subjected to a consolidation thermal treatment, consisting of $1 \mathrm{~h}$ at $60{ }^{\circ} \mathrm{C}, 1 \mathrm{~h}$ at $130{ }^{\circ} \mathrm{C}$, and calcined at $350{ }^{\circ} \mathrm{C}$ for $2 \mathrm{~h}$ (temperature ramp: $1{ }^{\circ} \mathrm{C} / \mathrm{min}$ ) to remove the templating agent. Finally, the samples were submerged in a wet etching solution, which is selective to the cooper layer comprised of $\mathrm{HCl} / \mathrm{H}_{2} \mathrm{O}_{2} / \mathrm{H}_{2} \mathrm{O}$ in a 1:1:5 ratio for $30 \mathrm{~min}$ at $60{ }^{\circ} \mathrm{C}$. Figure S1 (Supporting Information) shows a schematic diagram of these fabrication steps to obtain the patterned mesoporous films.

Micrographs were obtained using a Zeiss LEO 982 Gemini field-emission electron microscope in the secondary electron mode, using an in-lens detector to improve resolution.

In order to control substrate temperature, it was brought into contact with a thermoelectric cell (SF-COM-10080, SparkFun Electronics) connected to an $I-V$ source measure unit Keithley 2612A, and temperature values were acquired with a resistance temperature detector (Pt100) in contact with the sample. All experiments were carried out at $25{ }^{\circ} \mathrm{C}$ of room temperature and $46 \% \mathrm{RH}$ by placing $2 \mu \mathrm{L}$ drops. The cooling plate was in close contact with the silicon substrate, and no thermal gradients were observed in the region of interest. Images were recorded using a Mitutoyo FS70 microscope with a high-resolution digital camera.

\section{ASSOCIATED CONTENT}

\section{(5) Supporting Information}

The Supporting Information is available free of charge on the ACS Publications website at DOI: 10.1021/acsomega.8b02376.

File with Figures S1-S3 (PDF)

Movie S1: Dynamics of directional water collection (AVI)

\section{AUTHOR INFORMATION}

\section{Corresponding Authors}

*E-mail: mbellino@cnea.gov.ar (M.G.B.).

*E-mail: cberli@santafe-conicet.gov.ar (C.L.A.B.).

\section{ORCID}

Martín Gonzalo Bellino: 0000-0002-6713-6688 Claudio Luis Alberto Berli: 0000-0002-1321-6738

\section{Notes}

The authors declare no competing financial interest.

\section{ACKNOWLEDGMENTS}

R.G. acknowledges the doctoral fellowships from CONICET, Argentina. This work was supported by CONICET (PIP0363) and ANPCyT (PICT-2016-1781), Argentina.

\section{REFERENCES}

(1) Sun, T.; Feng, L.; Gao, X.; Jiang, L. Bioinspired surfaces with special wettability. Acc. Chem. Res. 2005, 38, 644-652.

(2) Parker, A. R.; Lawrence, C. R. Water capture by a desert beetle. Nature 2001, 414, 33-34.

(3) Ju, J.; Bai, H.; Zheng, Y.; Zhao, T.; Fang, R.; Jiang, L. A multistructural and multi-functional integrated fog collection system in cactus. Nat. Commun. 2012, 3, 1247.

(4) Bearzotti, A.; Bertolo, J. M.; Innocenzi, P.; Falcaro, P.; Traversa, E. Humidity sensors based on mesoporous silica thin films synthesised by block copolymers. J. Eur. Ceram. Soc. 2004, 24, 1969-1972.

(5) Innocenzi, P.; Martucci, A.; Guglielmi, M.; Bearzotti, A.; Traversa, E. Electrical and structural characterisation of mesoporous silica thin films as humidity sensors. Sens. Actuators, B 2001, 76, 299303.

(6) Boissiere, C.; Grosso, D.; Lepoutre, S.; Nicole, L.; Bruneau, A. B.; Sanchez, C. Porosity and mechanical properties of mesoporous thin films assessed by environmental ellipsometricporosimetry. Langmuir 2005, 21, 12362-12371.

(7) Mercuri, M.; Pierpauli, K.; Bellino, M. G.; Berli, C. L. A. Complex Filling Dynamics in Mesoporous Thin Films. Langmuir 2016, 33, 152-157.

(8) Berli, C. L. A.; Mercuri, M.; Bellino, M. G. Modeling the abnormally slow infiltration rate in mesoporous films. Phys. Chem. Chem. Phys. 2017, 19, 1731-1734.

(9) Mercuri, M.; Pierpauli, K. A.; Berli, C. L. A.; Bellino, M. G. An Open Pit Nanofluidic Tool: Localized Chemistry Assisted by Mesoporous Thin Film Infiltration. ACS Appl. Mater. Interfaces 2017, 9, 16679-16684.

(10) Mercuri, M.; Berli, C. L. A.; Bellino, M. G. Mesoporous Thin Films for Fluid Manipulation. Adv. Mater. Interfaces 2017, 4, 1700970.

(11) Gimenez, R.; Delgado, D. C.; Palumbo, F.; Berli, C. L. A.; Bellino, M. G. Mesoporous metal-oxide-semiconductor capacitors detect intra-porous fluid changes. Colloids Surf., A 2017, 524, 66-70.

(12) Gimenez, R.; Mercuri, M.; Berli, C. L. A.; Bellino, M. G. Electrical current nanogeneration driven by spontaneous nanofluidic oscillations. Nanoscale 2018, 10, 3144-3147.

(13) Innocenzi, P.; Kidchob, T.; Falcaro, P.; Takahashi, M. Patterning techniques for mesostructured films. Chem. Mater. 2008, 20, 607-614.

(14) Hu, H.; Larson, R. G. Evaporation of a sessile droplet on a substrate. J. Phys. Chem. B 2002, 106, 1334-1344.

(15) Erbil, H. Y. Evaporation of pure liquid sessile and spherical suspended drops: A review. Adv. Colloid Interface Sci. 2012, 170, 6786.

(16) Crank, J. The Mathematics of Diffusion, 2nd ed.; Clarendon Press, 1975.

(17) Cira, N. J.; Benusiglio, A.; Prakash, M. Vapour-mediated sensing and motility in two-component droplets. Nature 2015, 519, 446-450.

(18) Sáenz, P. J.; Wray, A. W.; Che, Z.; Matar, O. K.; Valluri, P.; Kim, J.; Sefiane, K. Dynamics and universal scaling law in geometrically-controlled sessile drop evaporation. Nat. Commun. 2017, 8, 14783

(19) Vincent, O.; Marguet, B.; Stroock, A. D. Imbibition triggered by capillary condensation in nanopores. Langmuir 2017, 33, 1655-1661. 
(20) Israelachvili, J. N. Intermolecular and Surface Forces; Academic press, 2011.

(21) CRC Handbook of Chemistry and Physics, 85th ed.; Lide, D. R., Ed.; CRC Press, 2004.

(22) Bass, J. D.; Grosso, D.; Boissiere, C.; Belamie, E.; Coradin, T.; Sanchez, C. Stability of Mesoporous Oxide and Mixed Metal Oxide Materials under Biologically Relevant Conditions. Chem. Mater. 2007, 19, 4349-4356.

(23) Zangooie, S.; Bjorklund, R.; Arwin, H. Water interaction with thermally oxidized porous silicon layers. J. Electrochem. Soc. 1997, 144, 4027-4035.

(24) Kim, H.; Rao, S. R.; Kapustin, E. A.; Zhao, L.; Yang, S.; Yaghi, O. M.; Wang, E. N. Adsorption-based atmospheric water harvesting device for arid climates. Nat. Commun. 2018, 9, 1191. 